\title{
Isolated Renal Mucormycosis in a Pediatric Patient
}

\section{JCR}

Parul Sobti, Garima Rakheja, Ashna Mittal, Nita Khurana, SK Aggarwal'

From the Department of Pathology and Paediatric Surgery', Maulana Azad Medical

College, Bahadur Shah Zafar Marg, New Delhi: 1 10002, India.

\begin{abstract}
:
Mucormycosis is an opportunistic fungal infection that rarely occurs in immunocompetent individuals. Mucormycosis refers to a serious fungal infection caused by fungi of the order Mucorales. The common clinical presentations of mucormycosis are rhinocerebral, cutaneous, pulmonary, gastrointestinal and disseminated forms. Clinically, most patients with isolated renal mucormycosis present with fever, flank pain, tenderness, hematuria or pyuria. Histopathologic evidence of fungal invasion of the tissues is diagnostic. Early institution of amphotericin- $B$, combined with nephrectomy offers the best chance for cure. Isolated renal mucormycosis has been seldom reported and is even rarer in children. We present an unusual case of renal mucormycosis in a pediatric patient with no known predisposing factors.
\end{abstract}

Keywords: Mucormycosis, Mycoses, Hematuria, Flank Pain, Kidney, Nephrectomy.

\section{Introduction}

Mucormycosis is an opportunistic fungal infection that rarely occurs in immunocompetent individuals. Involvement of a single organ such as bone, heart and kidney is extremely rare. Isolated renal mucormycosis has been seldom reported and is even rarer in children [1-3]. We present an unusual case of renal mucormycosis in a pediatric patient with no known predisposing factors [4].

\section{Case Report}

A four year old female presented with history of decreased appetite, continuous left loin pain, fever and episodes of vomiting for one week. On examination, tenderness was present in the left lumbar and left iliac region. Urine and blood culture showed no growth. Serological tests for human immunodeficiency virus, Hepatitis B and Hepatitis $C$ were negative. Ultrasound examination revealed a normal right kidney, and an enlarged left kidney, with scattered, ill-defined hypoechogenic areas with blurring of costal margin suggestive of acute pyelonephritis. Intravenous urography [Fig.1A] showed a non- functioning left kidney, with rest of the urinary tract being normal. CECT whole abdomen showed an enlarged left kidney, with non-homogenous attenuation and multiple varying sized areas of low attenuation partially replacing the normal parenchyma [Fig.1B]. The normal renal parenchyma appeared unorganized

\section{Corresponding Author: Dr. Parul Sobti}

Email: sobti.parul@gmail.com

Received: June 12,2013 | Accepted: September 13, 2013 | Published Online: October 30, 2013

This is an Open Access article distributed under the terms of the Creative Commons Attribution License (creativecommons.org/licenses/by/3.0)

Conflict of interest: None declared | Source of funding: Nil | DOl: http://dx.doi.org/10.17659/01.2013.0090 
with patchy areas of normal renal tissue scattered in the renal fossa. The right kidney was normal in size, outline, position and attenuation. A provisional clinical diagnosis of pyelonephritis (probably tubercular) was considered. The patient was started on ceftriaxone, metronidazole and amikacin. Nephrectomy revealed left kidney covered by exudate and measuring $8 \times 4 \times 4 \mathrm{~cm}$ [Fig. 1C]. On cut section, congestion with large areas of necrosis and multiple areas of abscess formation were seen. Microscopic examination revealed large abscesses replacing the renal parenchyma with extensive caseation and extensive areas of necrosis [Fig.2A,B] with broad aseptate fungal hyphae showing right angled branching [Fig. $2 \mathrm{C}$ ]. Angioinvasion and areas of renal infarction were also noted [Fig.2D].

The patient was started on amphotericin B

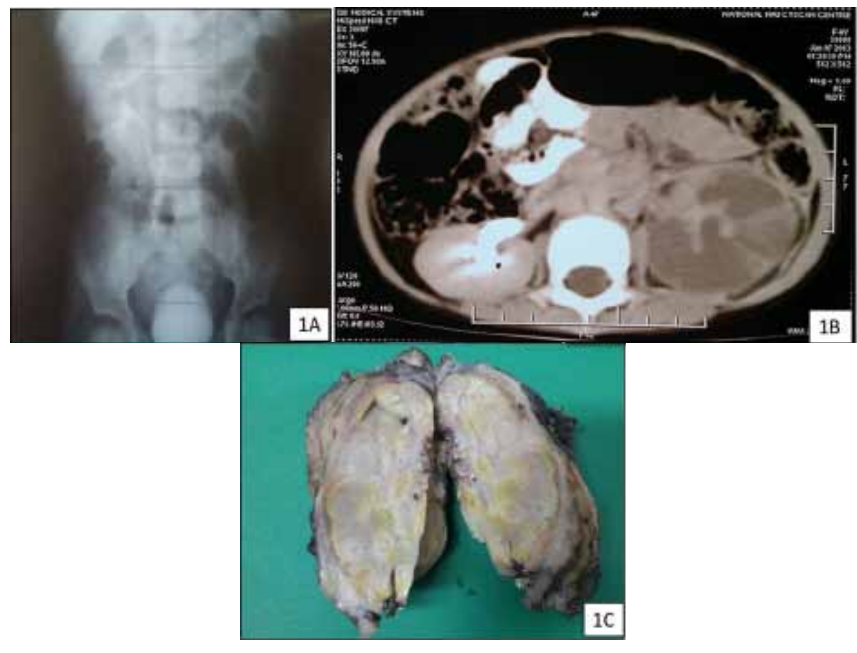

Fig.1A: Intravenous urography: left kidney impaired; right kidney normal. Fig.1 B: Left kidney: enlarged, with multiple varying sized areas of low attenuation partially replacing the normal parenchyma. The normal renal parenchyma appears unorganized, with patchy areas of normal renal tissue scattered in the renal fossa. Right kidney: Normal. Fig.1 C: Grossly, left kidney appeared congested, covered with exudate withfew necrotic areas present near the hilum. subsequent to the diagnosis and responded well to therapy. The patient is asymptomatic on 6 months of follow up.

\section{Discussion}

Mucormycosis refers to a serious fungal infection caused by fungi of the order Mucorales and genera Rhizopus, Absidia and Mucor [5]. Neutrophil and macrophage function is crucial for host defense against mucormycosis [6]. Thus patients with neutrophil or macrophage dysfunction have a higher susceptibility to develop this condition. Characteristically, patients with mucormycosis have extensive angioinvasion with vessel thrombosis, necrosis and penetration through lining of blood vessels leading to hematogenous dissemination. There is direct invasion of blood vessels and renal parenchyma. Renal infarction as seen in our case is due to total occlusion of the renal vessels by fungal hyphae.

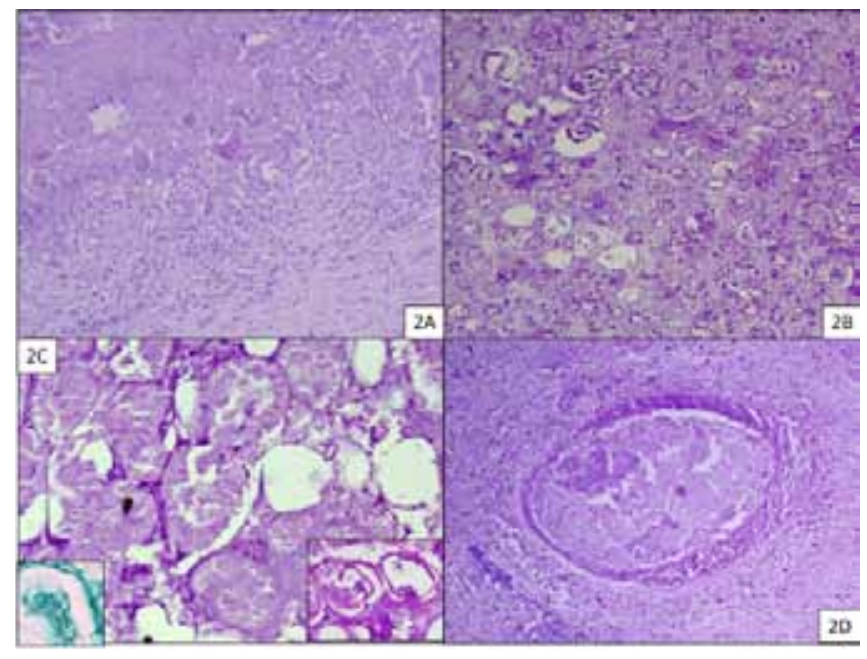

Fig.2A: Multiple epithelioid cell granulomas surrounding areas of necrosis (H\&E, 100X). Fig.2B: Renal infarction (H\&E, 100X). Fig.2C: Broad septate fungal hyphae with right angled branching (H\&E, 600X); Fungi positive on PAS and silver methanamine stain (inset, 1000X). Fig.2D: Renal vein thrombosis (H\&E, 100X). 
The common clinical presentations of mucormycosis are rhinocerebral, cutaneous, pulmonary, gastrointestinal and disseminated forms. Mode of infection is by inhalation or by ingestion and is severe and fatal except for the cutaneous form. The organism undergoes wide dissemination throughout the body due to its tendency for angioinvasion. The disseminated form constitutes $9 \%$ of cases of mucormycosis. Involvement of the kidneys has been reported in up to $20 \%$ of the cases with disseminated forms [7]. Involvement of a single organ such as bone, kidney and heart is extremely rare $[8,9]$. Overall survival for isolated renal mucormycosis is $65 \%$ [10].

Clinically, most patients with isolated renal mucormycosis present with fever, flank pain, tenderness, hematuria or pyuria. Blood and urine cultures rarely yield any organism and diagnosing mucormycosis almost always requires a high index of clinical suspicion. Histopathological identification of fungal hyphae remains the gold standard for diagnosis. The biopsy reveals broad aseptate, thin walled hyphae with right angled branching. The thin wall leads to weak staining with Gomori methanamine silver and Periodic Acid Schiff.

Isolated Renal Mucormycosis is a rare entity, but, has an overall favorable outcome comparedto other forms of invasive mucormycosis. The paradoxical combination of benign clinical findings (indolent course with benign urine or blood culture) and worrisome radiographic findings (evidence of severe localized pyelonephritis) should alert one to consider renal infection with an angioinvasive organism. Histopathologic evidence of fungal invasion of the tissues is diagnostic. Early institution of amphotericin- $B$, combined with nephrectomy offers the best chance for cure.

\section{References}

1. Raghavan R, Date A, Bhaktaviziam A. Fungal and nocardial infections of the kidney. Histopathology.1987;1 1:9-20.

2. Davila RM, Moser SA, Grosso LE. Renal mucormycosis: A case report and review of the literature. J Urol. 1991;145:1242-1244.

3. Pickles R, Long $G$, Murugasu R. Isolated renal mucormycosis. Med J Aust. 1994; 160:514-516.

4. Yun-Sun $H$, Singh N. Mucormycosis: its contemporary face and management stratégies. Lancet Infect Dis. $2011 ; 11: 301-311$.

5. Gupta KL. Fungal Infections and the kidney. Indian J Nephrol. 2001;1 1:147-154.

6. Ingram CW, Sennesh J, Cooper JN, Perfect JR. Disseminated zygomycosis: Report of four cases and review. Rev Infect Dis. 1989; 1 1:741-754.

7. Waldorf AR. Host-parasite relationship in opportunistic mycoses. Crit Rev Microbiol. 1986;13:133-172.

8. Gupta KL, Joshi K, Sud K, Kohli HS, Jha V, Radotra BD, et al. Renal zygomycosis: An underdiagnosed cause of acute renal failure. Nephrol Dial Transplant. 1999; 14:2720-2725.

9. Prout GR, Jr, Goddard R. Renal mucormycosis: Survival after nephrectomy and amphotericin $B$ therapy. N Engl J Med. 1960;263:1 246-1 248.

10. Sharma R, Shivanand G, Kumar R, Prem $S$, Kandpal H, Das J, et al. Isolated renal mucormycosis: an unusual cause of acuterenal infarction in a boy with aplastic anaemia. $\mathrm{Br} J$ Radiol. 2006;79:e19-21. 